\title{
Non-Commutative Geometry in Higher Dimensional Quantum Hall Effect as A-Class Topological Insulator
}

\author{
Kazuki Hasebe* \\ ${ }^{1}$ Kagawa National College of Technology, Takuma-cho, Mitoyo, Kagawa 769-1192, Japan \\ Received Date Month 2014, revised Date Month 2014, accepted Date Month 2014 \\ Published online Date Month 2014
}

Key words Quantum Nambu geometry, topological insulator, quantum Hall effect, monopole

We clarify relations between the higher dimensional quantum Hall effect and A-class topological insulator. In particular, we elucidate physical implications of the higher dimensional non-commutative geometry in the context of A-class topological insulator. This presentation is based on [1].

Copyright line will be provided by the publisher

\section{Introduction}

The non-commutative geometry (NCG) is the underlying geometry of the quantum Hall effect (QHE). In the lowest Landau level (LLL), the electron coordinates are identified with the center of mass coordinates that satisfy the $\mathrm{NC}$ algebra:

$$
[X, Y]=i \ell^{2} .
$$

Almost a decade ago, the time-reversal symmetric counterpart of QHE, dubbed as quantum spin Hall effect, was theoretically proposed [2] and experimentally confirmed [3]. Subsequently, the 3D version of the quantum spin Hall effect, topological insulator (TI), was also discovered [4]. Now we understand there exist a variety of topological classes of QHE with different symmetries as summarized in the topological periodic table [5]. One may wonder what kind of geometry will describe such TIs.

Recently two groups, [6] and [7], independently proposed the quantum Nambu bracket [8] for higher dimensional topological insulators. In Ref.[6], the authors considered the quantum Nambu 3-bracket for chiral TIs (AIII-class). Meanwhile the authors in Ref.[7] adopted the the even dimensional quantum Nambubracket for A-class TIs, and 3-bracket for 3D TI.

Inspired by the recent developments, we discuss A-class TIs with emphasis on their relation to higher D. QHE and NCG. The A-class TIs live in arbitrary even dimensional spaces and do not respect any symmetries such as time-reversal, particle-hole, and chiral symmetries like QHE. Indeed QHE is a 2D entity of A-class TIs, and the A-class TIs can be regarded as higher D counterparts of the QHE, i.e. higher D. QHE. The higher D. QHE was first constructed by Zhang and Hu in 4D [9] as a natural generalization of 2D QHE on Haldane's sphere [10]. Their 4D model was soon generalized in even higher dimensions[11, [12], such as complex projective spaces and even dimensional spheres. In the modern point of view, the higher D. QHE can be considered as a realization of the A-class TI with Landau levels. NCG naturally fits in even dimensional space since each commutator needs a pair of $\mathrm{NC}$ coordinates, and so all of the even dimensional coordinates of A-class TIs are neatly fitted in the commutators and NCG is realized in the whole space. Thus, A-class TIs are a good starting point to see what physical implications the higher D. NCG brings. We will discuss the spherical higher D. A-class TIs [1] on the basis of the former works [12].

* Corresponding author E-mail: hasebe@dg.kagawa-nct.ac.jp, Phone: +81 87583 8528, Fax: +81 875836389 


\section{Non-Commutative Geometry of Higher Dimensional Fuzzy Sphere}

The coordinates of fuzzy two-sphere, $S_{F}^{2}$, are defined so as to satisfy the $S U(2)$ algebra [13]:

$$
\left[X_{i}, X_{j}\right]=i \alpha \epsilon_{i j k} X_{k}, \quad(i, j, k=1,2,3)
$$

where $\alpha=2 r / I$. (I/2 corresponds to the $S U(2)$ spin magnitude.) In general, the coordinates of $2 k \mathrm{D}$ fuzzy sphere $S_{F}^{2 k}$ satisfy [14]

$$
\left[X_{a}, X_{b}\right]=i \alpha X_{a b}, \quad(a, b=1,2, \cdots, 2 k+1)
$$

where $X_{a b}$ denote the $S O(2 k)$ operators. For 3D, the 2rank tensor is equivalent to the vector by the relation $X_{i j}=\epsilon_{i j k} X_{k}$, and then (2) is realized as a special case of (3). Except for the special 3D case $(k=1)$, Eq. (3) is not closed only by $X_{a}$ themselves, but the extra operators $X_{a b}$ are introduced on the right-hand side. There is another mathematical formulation for $S_{F}^{2 k}$ by using the Nambu bracket [15]:

$$
\left[X_{a_{1}}, X_{a_{2}}, \cdots, X_{a_{2 k}}\right]=i^{k} C(k, I) \alpha^{2 k-1} \epsilon_{a_{1} a_{2} \cdots a_{2 k+1}} X_{a_{2 k+1}},
$$

where $C(k, I)=\frac{(2 k) ! !(I+2 k-2) ! !}{2^{2 k-1} I ! !}$ and $\left[X_{a_{1}}, X_{a_{2}}, \cdots, X_{a_{2 k}}\right] \equiv X_{\left[a_{1}\right.}, X_{a_{2}}, \cdots, X_{\left.a_{2 k}\right]}$. Eq.(4) is more elegant than Eq.(3) in the sense that the $S O(2 k)$ operators do not apparently appear the algebra. Thus, there are two superficially different mathematical formulations for higher D. fuzzy sphere.

A simplest way to see the corresponding monopole set-up is to find the right-hand side of the NC algebra. From $S_{F}^{2}$ (2), we can read off the Dirac monopole field strength:

$$
F_{i} \simeq \frac{1}{r^{3}} x_{i} . \quad(i=1,2,3)
$$

Similarly from (3] and (4), we obtain the $S O(2 k)$ non-abelian monopole field strength [16] and the $U(1)$ tensor monopole field strength [16]:

$$
\begin{aligned}
& F_{\mu \nu} \simeq \frac{1}{r^{2}} \Sigma_{\mu \nu}, \quad(\mu, \nu=1,2, \cdots, 2 k) \\
& G_{a_{1} a_{2} \cdots a_{2 k}} \simeq \frac{1}{r^{2 k+1}} \epsilon_{a_{1} a_{2} \cdots a_{2 k+1}} x_{a_{2 k+1}}, \quad\left(a_{1}, a_{2}, \cdots, a_{2 k}=1,2, \cdots, 2 k+1\right)
\end{aligned}
$$

where $\Sigma_{\mu \nu}$ denote the $S O(2 k)$ matrices.

\section{Non-Abelian and Tensor Monopole Correspondence}

Corresponding to the two different $\mathrm{NC}$ formulations, we obtain two different monopole set-ups. However, since the two NC formulations describe the same NC manifold, i.e. $S_{F}^{2 k}$, the corresponding two monopole set-ups should be same in some sense. Indeed there exists a simple correspondence:

$$
G_{2 k}=\operatorname{tr}\left(F^{2 k}\right)
$$

For the fully symmetric representation $[I / 2, I / 2, \cdots, I / 2]$, the right-hand side of (7) gives

$$
\operatorname{tr}\left(F^{2 k}\right)=\frac{1}{2^{k+1} r^{2 k+1}} c_{k}(I) \epsilon_{a_{1} a_{2} \cdots a_{2 k+1}} x_{a_{2 k+1}} d x_{a_{1}} d x_{a_{2}} \cdots d x_{a_{2 k}},
$$

where $c_{k}(I)=\prod_{l=1}^{k} \prod_{i=1}^{l} \frac{I+l+i-2}{l+i-1}$ denotes the $k$ th Chern-number of the $S O(2 k)$ monopole on $S^{2 k}$. Eq. (8) is actually equivalent to the tensor monopole field strength:

$$
G_{2 k}=g_{k} \frac{1}{(2 k) ! r^{2 k+1}} \epsilon_{a_{1} a_{2} \cdots a_{2 k+1}} x_{a_{2 k+1}} d x_{a_{1}} d x_{a_{2}} \cdots d x_{a_{2 k}},
$$


with tensor monopole charge $g_{k}=\frac{(2 k) !}{2^{k+1}} c_{k}(I)$. The monopole gauge fields are similarly related as

$$
C_{2 k-1}=\operatorname{tr}\left(L_{\mathrm{CS}}^{(2 k-1)}[A]\right),
$$

where $L_{\mathrm{CS}}^{2 k-1}[A]$ denotes the Chern-Simons action in $(2 k-1) \mathrm{D}$ :

$$
L_{\mathrm{CS}}^{(2 k-1)}[A]=k \int_{0}^{1} d t \operatorname{tr}\left(A\left(t d A+i t^{2} A^{2}\right)^{k-1}\right) .
$$

For instance, $L_{\mathrm{CS}}^{(1)}[A]=\operatorname{tr} A, L_{\mathrm{CS}}^{(3)}[A]=\operatorname{tr}\left(A d A+\frac{2}{3} i A^{3}\right)$ and $L_{\mathrm{CS}}^{(5)}[A]=\operatorname{tr}\left(A(d A)^{2}+\frac{3}{2} i A^{3} d A-\frac{3}{5} A^{5}\right)$. By substituting the $S O(2 k)$ gauge field to the right-hand side of $[10)$, we have

$$
\begin{aligned}
& C_{1}=-c_{1}(I) \frac{1}{2 r\left(r+x_{3}\right)} \epsilon_{i j 3} x_{j} d x_{i}, \\
& C_{3}=-c_{2}(I) \frac{1}{6 r^{3}}\left(\frac{1}{r+x_{5}}+\frac{r}{\left(r+x_{5}\right)^{2}}\right) \epsilon_{a b c d 5} x_{d} d x_{a} d x_{b} d x_{c}, \\
& C_{5}=-c_{3}(I) \frac{3}{40 r^{5}}\left(\frac{1}{r+x_{7}}+\frac{r}{\left(r+x_{7}\right)^{2}}+\frac{2}{3} \frac{r^{2}}{\left(r+x_{7}\right)^{3}}\right) \epsilon_{a b c d e f 7} x_{f} d x_{a} d x_{b} d x_{c} d x_{d} d x_{e} .
\end{aligned}
$$

$C_{3}$ and $C_{5}$ represent a natural generalization of the Dirac monopole gauge field $C_{1}$. In general, the $(2 k-1)$ rank tensor monopole gauge field exhibits the $k$ th power string-like singularity.

In the lowest Landau level (LLL), the covariant angular momentum vanishes and so the total angular momentum is identified with the field strength:

$$
L_{a b}=\Lambda_{a b}+r^{2} F_{a b} \sim r^{2} F_{a b} .
$$

According to 17, we have $\frac{1}{r^{2 k+1}} x_{a}=\frac{2}{(2 k) ! c_{k}(I)} \epsilon_{a a_{1} a_{2} \cdots a_{2 k}} \operatorname{tr}\left(F_{a_{1} a_{2}} \cdots F_{a_{2 k-1} a_{2 k}}\right)$, and with (13), we find that the coordinates in the LLL become to

$$
X_{a}=\frac{I}{(2 k) ! c_{k}(I)} \alpha \epsilon_{a a_{1} a_{2} \cdots a_{2 k}}\left(L_{a_{1} a_{2}} L_{a_{3} a_{4}} \cdots L_{a_{2 k-1} a_{2 k}}\right),
$$

which obey the quantum Nambu algebra (4). Thus, the quantum Nambu geometry naturally emergies in the LLL of A-class TIs.

\section{Tensor Chern-Simons Theory for A-Class Topological Insulator}

The non-Abelian and tensor monopole correspondence brings important physical implications to the A-class topological insulators. In the original QHE, the Chern-Simons (CS) flux attachment is introduced to cancel the external $U(1)$ magnetic field:

$$
A_{1}-C_{1}=0 .
$$

This cancellation is rather trivial in the sense both external and Chern-Simons gauge fields are Abelian. In higher dimensions, the external magnetic fields are non-Abelian gauge fields. The non-Abelian and tensor monopole correspondence tells that the non-abelian magnetic field can be canceled by Abelian gauge field:

$$
\operatorname{tr}\left(L_{\mathrm{CS}}^{(2 k-1)}[A]\right)-C_{2 k-1}=0 .
$$

For instance $k=2,3$, we have

$$
\begin{aligned}
& \operatorname{tr}\left(A d A+\frac{2}{3} i A^{3}\right)-C_{3}=0 \\
& \operatorname{tr}\left(A(d A)^{2}+\frac{3}{2} i A^{3} d A-\frac{3}{5} A^{5}\right)-C_{5}=0 .
\end{aligned}
$$


Due to this notable cancellation, we can generalize exotic ideas cultivated in the QHE to A-class TIs, such as topological field theory description of QHE [18]. We propose the following tensor-type Chern-Simons field theory for the A-class TIs:

$$
S=\int_{4 k-1} C_{2 k-1} J_{2 k}+\frac{1}{4 \pi m^{k}} \int_{4 k-1} C_{2 k-1} G_{2 k},
$$

where $G_{2 k}=d C_{2 k-1}$ and $m$ denotes an odd integer in accordance with the filling factor $\nu_{2 k}=1 / \mathrm{m}^{k}$. The membranes described by the tensor Chern-Simons action (18) generally obey the fractional statistics related the linking number [21]. The equations of motion of the Chern-Simons field yield

$$
J_{2 k}=-\frac{1}{2 \pi m^{k}} G_{2 k}
$$

For component representation, Eq. 196) can be rewritten as

$$
\begin{aligned}
& J^{i_{1} i_{2} \cdots i_{2 k-2} 0}=-\frac{1}{2 \pi m^{k}} B^{i_{i} i_{2} \cdots i_{2 k-2}}, \\
& J^{i_{1} i_{2} \cdots i_{2 k-1}}=\frac{1}{2 \pi(2 k) ! m^{k}} \epsilon^{i_{1} i_{2} \cdots i_{4 k-2}} E_{i_{2 k} \cdots i_{4 k-2}},
\end{aligned}
$$

Eqs. (20) represent generalized flux attachment and Hall effect. The $(2 k-1)$-form Chern-Simons field is naturally coupled to $(2 k-2)$ dimensional membrane-like object, $(2 k-2)$-brane. Since $m^{k}$ is an odd integer, the odd number fluxes are attached to membrane and the membrane becomes a composite object of the original membrane and the fluxes. Since at $\nu_{2 k}=1$, membrane corresponds to a fermion, the odd number $m^{k}$ flux attachment transmutes the statistics of the membrane to Bose statistics just like composite boson. The groundstate of the A-class TI can be regarded as a superfluid state of the composite membranes. The fractionally charged quasi-particle in fractional QHE [19] is also generalized to $(2 k-2)$ brane excitation with a fractional charge:

$$
e_{2 k-2}^{*}=1 / m^{k} .
$$

It turns out that the fractional charged membrane obeys the fractional statistics mediated by the tensor gauge field. Particularly interesting property is the dimensional hierarchy. From the 0-brane picture, the total filling factor is expressed as

$$
\nu=\nu_{2} \nu_{4} \nu_{6} \cdots \nu_{2 k}=1 / m^{\frac{1}{2} k(k+1)} .
$$

The hierarchical structure of the filling factor may remind the Haldane-Halperin hierarchy of the fractional QHE [10, 20], however notice that the hierarchy (22) ranges over different dimensions not in the same dimension. According to the Haldane-Halperin's idea of the hierarchy, the hierarchical structure of the filling fraction implies the condensation of quasi-excitations to form a new generation of incompressible liquid. The dimensional hierarchy of the filling fraction implies that the low dimensional membrane-like objects condense to make a higher dimensional quantum incompressible liquid of A-class TIs.

\section{Summary}

We exploited the A-class TI with emphasis on its relations to quantum Nambu geometry. The main observations are summarized in Fig 1 . We showed that the exotic physics of QHE can naturally be generalized in A-class TIs based on the non-Abelian and tensor monopole correspondence. While we focused on the A-class TIs, similar discussions can be expanded for AIII-class TIs that live in arbitrary odd dimensional spaces [22]. 


\section{Lie algebraic commutator $\rightarrow$ Colored monopole $\rightarrow$ Higher D. QHE $\uparrow$ Fuzzy sphere $\quad \uparrow \begin{aligned} & \text { Physical } \\ & \text { realization }\end{aligned}$}

\section{Quantum Nambu algebra $\rightarrow$ Tensor monopole $\rightarrow$ A-class TI}

Fig. 1 The fuzzy sphere has two superficially different mathematical formulations. Corresponding to the two realizations, there are two monopole set-ups related by the Chern-Simons action. Higher D. QHE is a realization of the A-class TI with Landau levels.

Acknowledgements This work was partially supported by a Grant-in-Aid for Scientific Research from the Ministry of Education, Science, Sports and Culture of Japan (Grant No.23740212), Overseas Dispatching Program 2013 of National College of Technology, and The Emma Project for Art and Culture.

\section{References}

[1] Kazuki Hasebe, Nucl. Phys. B 886 (2014) 952-1002.

[2] C.L. Kane, E.J. Mele, Phys. Rev. Lett. 95 (2005) 146802. B. Andrei Bernevig, Shou-Cheng Zhang, Phys. Rev. Lett. 96 (2006) 106802. B. Andrei Bernevig, Taylor L. Hughes, Shou-Cheng Zhang, Science, 314 (2006) 1757.

[3] Markus Koenig et al, Science, 318 (2007) 766.

[4] Liang Fu and C. L. Kane, Phys. Rev. B 76 (2007) 045302. Shuichi Murakami, New Journal of Physics 9 (9): 356. D. Hsieh, et al. Nature 452 (9): 970-974.

[5] Andreas P. Schnyder, et al. Phys. Rev. B 78 (2008) 195125. A. Kitaev, arXiv:0901.2686 Shinsei Ryu, et al. New J. Phys. 12 (2010) 065010.

[6] Titus Neupert, et al. Phys. Rev. B 86 (2012) 035125.

[7] B. Estienne, N. Regnault, B. A. Bernevig, Phys. Rev. B 86 (2012) 241104(R).

[8] Yoichiro Nambu, Phys.Rev.D7 (1973) 2405-2412. Thomas Curtright, Cosmas Zachos, Phys.Rev.D68 (2003) 085001.

[9] S. C. Zhang and J. P. Hu, Science 294 (2001) 823.

[10] F.D.M. Haldane, Phys. Rev. Lett. 51 (1983) 605-608.

[11] D. Karabali, V. P. Nair, Nucl.Phys. B641 (2002) 533. Brian P. Dolan, JHEP 0305 (2003) 018. B. A. Bernevig, J. P. Hu, N. Toumbas, S. C. Zhang, Phys.Rev.Lett. 91 (2003) 236803.

[12] K. Hasebe and Y. Kimura, Phys.Lett. B 602 (2004) 255. Kazuki Hasebe, SIGMA 6 (2010) 071.

[13] F.A. Berezin, Commun.Math. Phys. 40 (1975) 153-174. J. Hoppe, MIT PhD Thesis (1982). J. Madore, Class. Quant. Grav. 9 (1992) 69.

[14] H. Grosse, C. Klimcik, P. Presnajder, Commun.Math.Phys. 180 (1996) 429-438. D. Kabat, W. Taylor, Adv.Theor.Math.Phys.2 (1998) 181-206. S. Ramgoolam, Nucl.Phys. B610 (2001) 461-488. P. M. Ho and S. Ramgoolam, Nucl.Phys.B 627 (2002) 266. Yusuke Kimura, Nucl.Phys. B637 (2002) 177-198. Nucl.Phys. B664 (2003) 512-530.

[15] M. M. Sheikh-Jabbari, JHEP 0409 (2004) 017. M. M. Sheikh-Jabbari, M. Torabian, JHEP 0504 (2005) 001. Joshua DeBellis, Christian Saemann, Richard J. Szabo, J.Math.Phys.51 (2010) 122303.

[16] Chen Ning Yang, J. Math. Phys. 19 (1978) 320. Z. Horvath and L. Palla, Nucl.Phys. B142 (1978) 327. D. H. Tchrakian, J.Math. Phys. 21 (1980) 166. Bernard Grossman, et al. Commun.Math.Phys. 96 (1984) 431, Erratumibid.100 (1985) 311. C. Saclioglu, Nucl.Phys. B277 (1986) 487.

[17] R.I. Nepomechie, Phys. Rev. D 31 (1985) 1921. Claudio Teitelboim, Phys.Lett. B 167 (1986) 63-68, Phys.Lett. B 167 (1986) 69-72.

[18] S.C. Zhang, T.H. Hansson, S. Kivelson Phys.Rev.Lett.62 (1988) 82-85. S.C. Zhang, Int.J.Mod.Phys. B6 (1992) 25-58.

[19] D. Arovas, J. R. Schrieffer, F. Wilczek, Phys.Rev. Lett. 53 (1984) 722.

[20] B. I. Halperin, Phys. Rev. Lett. 52 (1984) 1583-1586.

[21] R.I. Nepomechie and A. Zee, Quantum Field Theory and Quantum Statistics, 2 (1984) 467-472. Y.S. Wu, A. Zee, Phys. Lett. B 207 (1988) 39. C-H Tze, S. Nam, Annals of Phys. 193 (1989) 419.

[22] Kazuki Hasebe, Nucl. Phys. B 886 (2014) 681-690. 